\title{
Transitioning to nursing practice in Lebanon: Challenges in professional, occupational and cultural identity formation
}

\author{
Murielle Madi*1, Michael Clinton ${ }^{2}$, Myrna Doumit $^{3}$, Sawsan Ezzeddine ${ }^{4}$, Ursula Rizk ${ }^{5}$ \\ ${ }^{1}$ American University of Beirut Medical Center, Beirut, Lebanon \\ ${ }^{2}$ Hariri School of Nursing, American University of Beirut, Lebanon \\ ${ }^{3}$ Alice Ramez Chagouri School of Nursing, Lebanese American University, Byblos, Lebanon \\ ${ }^{4}$ College of Nursing and Health Sciences, Makassed University of Beirut, Lebanon \\ ${ }^{5}$ Nursing Program, University of Balamand, Lebanon
}

Received: October 27, 2017

DOI: $10.5430 /$ jnep.v8n6p38
Accepted: December 22, 2017

Online Published: January 2, 2018

\begin{abstract}
The aim of this study was to identify the challenges graduates from three of Lebanon's leading universities face as they transition from the role of student to first year registered nurse. Focus group discussions and one joint interview were conducted with 16 first year registered nurses transitioning to practice in university medical centers in Greater Beirut. Thematic analysis was used to summarize the challenges faced by the graduates. Initially, three descriptive themes were used to summarize the data: classroom learning, workplace realities, and "wanting a life". Together the three themes indicted that classroom instruction of baccalaureate nursing students in Lebanon raises expectations for ideal practice that cannot be realized in clinical units with high workloads and nursing shortages. As a result, first year registered nurses are made to feel unwelcome unless they compromise their values and adapt quickly to the pace of work. The three initial themes were revised deductively from the perspective of ego-identity theory to explain the relationship between transitioning to nursing practice and identity formation in late adolescence and early adulthood. If the pressures of identity formation are not addressed, first year registered nurses in Lebanon will be at risk for acquiescing to task-centered practice, abandoning bedside care for administrative roles, or leaving nursing. The evidence for this conclusion will interest nursing faculty, hospital administrators, nurse leaders, registered nurses, physicians, and nursing students.
\end{abstract}

Key Words: Focus groups, Thematic analysis, First year registered nurses, Transition to nursing practice, Ego-identity theory, Identity formation, Lebanon

\section{INTRODUCTION}

Graduating from nursing school and transitioning to practice are important turning points for first year registered nurses (FYRNs), but leaving the familiar and supportive environment of the classroom for the clinical setting is intimidating and can be overwhelming. Few new graduates make the transition without anxiety, self-doubt and second thoughts about a career in nursing. ${ }^{[1,2]}$ Although they have completed their nursing program FYRNs are still learning when they enter the hospital setting. ${ }^{[3-7]}$ As a result, the experience of transitioning to the nursing workforce while needing to consolidate prior learning has been portrayed as highly stress-

\footnotetext{
*Correspondence: Murielle Madi; Email: muriellemadi1@gmail.com; Address: American University of Beirut Medical Center, Riad El-Solh, Beirut,
} Lebanon. 
ful ${ }^{[8-10]}$ and full of stumbling blocks that have unpleasant repercussions for the professional and personal lives of new graduates. ${ }^{[3-5]}$ FYRNs need energy and courage to transition to nursing practice ${ }^{[11,12]}$ but success is not achieved easily and too many FYRNs become discouraged, disillusioned and ready to leave. ${ }^{[13]}$

The literature on the transition of nursing graduates to the role of registered nurse is varied and extensive, it is clear, that the FYRN is required to adapt quickly to a new and demanding clinical role. ${ }^{[10,13]}$ If they cannot adapt, FYRNs have invested in an expensive education they cannot capitalize on and the nursing workforce has lost the potential of a recruit at a time of concern about nursing shortages. As in the U.S, ${ }^{[14]}$ nursing shortages in Lebanon are affecting care processes, health care capacity and patient safety. ${ }^{[15,16]}$

Despite the many advances that have been made in nursing education, FYRNs are still struggling to adapt to the clinical setting as a place of work as well as of learning. ${ }^{[17]}$ The perceived unpreparedness of FYRNs for practice is a concern for hospital managers because new graduates make more errors. ${ }^{[12]}$ New graduates make errors because practice is fast-paced and FYRNs must adapt to new work settings while compensating for nursing shortages. ${ }^{[5,11,12,17]}$ Additional pressure on FYRNs comes from the struggle to reconcile personal beliefs about what nursing should be with the more pragmatic expectations of experienced registiered nurses. The result has been described as role ambiguity ${ }^{[17,18]}$ and reality shock; ${ }^{[19]}$ neither of which is now considered exceptional. Nursing schools socialize their students into expecting reality shock and work with them to develop protective strategies before graduation. ${ }^{[3,13,20]}$ Cultivating empowerment is one of the more common strategies nursing schools and healthcare systems use to prepare students and FYRNs for practice. ${ }^{[9,21,22]}$

Any deficiency in orientation when FYRNs start to practice has a negative impact on their confidence. ${ }^{[5,11]}$ The stress of poor orientation is increased in the absence of regular constructive feedback ${ }^{[7,13]}$ and no time for the FYRN to learn how to improve performance. ${ }^{[10,20,23]}$ Poor orientation makes FYRNs feel unwelcome and they interpret absence of feedback as a lack of support. ${ }^{[10]}$ The support of a preceptor who can help facilitate acceptance by the work team and promote feelings of inclusion is invaluable in smoothing the transition of the FYRN. Particularly important is access to a preceptor who can help the FYRN cope with a constantly heavy workload while trying to grasp the official and unofficial rules of the unit and manage everyday realities. ${ }^{\text {[24] }}$

The demands of coming to terms with the realities of nursing practice and the constant struggle to achieve work-life balance when effectively powerless ${ }^{[10,25]}$ makes FYRNs vulnerable to workplace pressure and bullying. ${ }^{[3]}$ Pressure and bullying leave the FYRN stressed, distracted, professionally unsatisfied, and more inclined to leave their job or abandon nursing as a career choice. ${ }^{[2,10]}$ Experienced nurses continually strive for power within more powerful healthcare teams and are often afraid to stand up to the demeaning attitudes and condescension of more powerful groups. ${ }^{[17,22]}$ Some experienced registered nurses respond by taking out their frustration on FYRNs and other less experienced nurses. ${ }^{[17]}$ Workplace violence, once hardly mentioned, is becoming, or has become, the new norm for many nurses. ${ }^{[26]}$

The challenges of transitioning to nursing practice have been discussed extensively in the literature. However, in Lebanon, the topic has not yet received sufficient attention. Therefore, we conducted a study of the students and graduates of four university schools of nursing in Beirut to identify local factors that make the transition to nursing practice unnecessarily difficult. In this article, we describe the experiences of FYRNs who graduated from three of the universities because the graduates of the fourth university could not get time off to attend. We follow Leong and Crossman ${ }^{[27]}$ in understanding transition to mean the speed with which the FYRN becomes comfortable in the work environment. We define FYRN literally as a nurse in the first year of practice following graduation with a baccalaureate degree in nursing.

\section{METHOD}

\subsection{Ethics approval and recruitment of participants}

The study was approved by the institutional review boards of the four participating universities. The participants were recruited by posting flyers at the universities and their affiliated medical centers. The contact details of members of the research team were given on the flyers. FYRNs contacted the research team member of their choice for more information. When contacted, research team members explained the study, answered any questions and confirmed the dates, times and locations of focus group discussions. Written informed consent was taken immediately prior to conducting the focus group discussions and the joint interview. To encourage open discussion and avoid perceptions of undue influence, the authors did not collect data from graduates from their own institutions. The authors constantly reminded the participants of the importance of anonymity and confidentiality. Anonymity was further safeguarded by conducting the focus group discussions and the joint interview at university venues unrelated to the work settings of the participants. In this article, we protect anonymity and maintain confidentiality by using pseudonyms beginning with "W" for women with "M" for men. The data were stored and analyzed on password 
protected computers in the university offices of the authors Only the authors and their research assistants had access to the recordings and transcripts. The first author was a Master of Science in Nursing student when the study was conducted.

\subsection{Focus group participation}

A total of 16 FYRNs participated in the study. Four focus groups were organized, one for the graduates of each of the participating universities and their affiliated medical centers. The focus group for graduates from one university was cancelled because the participants could not attend due to their heavy workloads. When only two participants (one women and one man) attended for one of the focus groups, the discussion was conducted as a joint interview. The two focus groups that were conducted were attended by nine and five participants respectively. Overall, women participants outnumbered male participants almost two to one (10 women, 6 men). The number of participants in the joint interview and in one of the focus groups was less than the six to twelve members recommended for productive discussion. ${ }^{[28]}$ However, we regard the ability of focus group participants to contribute to in-depth discussions as equally if not more important than the number of members in attendance. ${ }^{[29]} \mathrm{We}$ note that the setting from which we were unable to recruit FYRNs was that with the highest nursing workloads. The joint interview data were pooled with the focus group data prior to analysis.

\subsection{Conduct of focus group and joint interview}

Although the language of instruction at the participating universities is English, the first language of Lebanese students is Arabic. The focus groups were conducted in English or Arabic according to the preference of the members. The third author conducted the focus group with nine participants in English. The fourth author conducted the other focus group in Arabic. The second author is not fluent in Arabic so he conducted the joint interview in English. The focal question posed to all the participants was: What challenges are you facing in your first year of practice? The first author and two research assistants fluent in Arabic and English prepared verbatim transcripts from the digital recordings of the focus group discussions. The translations of the Arabic transcripts were validated independently by three bilingual members of the research team (the third, fourth and fifth authors). When all transcripts were available in English, they were read and coded independently by all five authors. Initial data codes were developed by the first, second and fourth author and later modified and verified at successive research team meetings. The research team met to discuss interpretations of the data and the analyses published here were reviewed and revised by all five authors.

\subsection{Data analysis}

Microsoft Word and Microsoft Excel were used to manage the data because the authors worked as a team and used software readily available at the four universities. The line number function in Word was used to identify lines in the data prior to line by line coding. Codes and their line numbers were copied into Excel to sort and aggregate data. We used thematic analysis to analyze the data because it is flexible, practical and summarizes data in ways that make sense to participants as well as to investigators. ${ }^{[30]}$ Unlike grounded theory, thematic analysis does not require construction of substantive and formal theories; although it can support deductive as well as inductive inference. It differs from phenomenology in that it does not presuppose a philosophical theory and is unlike critical discourse analysis because it does not deny human subjectivity.

All five authors listened to the audio recordings and read through the transcripts independently. The first two authors developed initial codes for the data that were later refined in team meetings. The codes were revised and collapsed into broader categories to summarize the data. Care was taken to include data from both focus groups and the joint interview. The first two authors selected statements to exemplify the categories. Trial and error was used until consensus was reached on which quotations best exemplified the data. The categories were then collapsed and grouped into descriptive themes that were refined by adding subthemes to group the exemplary statements. The exemplary statements were moved among themes and subthemes until consensus was achieved on the most logical structure for reporting the the study findings. We regarded the exemplarly statements extracted from the data as credible if they were supported by focus group members, as transferable if they were validated in both focus groups, and dependable if they were consistent with comments made in the joint interview.

As we grouped our data into descriptive themes, we realized that baccalaureate nurse education in Lebanon coincides with late adolescence and early adulthood and noted that the four participating universities rarely receive applications for admission to nursing programs from older applicants. Accordingly, we began to re-conceptualize our data from the perspective of the age-related challenges relevant to young people in Lebanon. This led us to recall Erikson's ${ }^{[31,32]}$ crises in ego identity formation as taken up by Marcia in his discussion of ego-identity, occupational choice, and occupational commitment. ${ }^{[33]}$ Although this literature is dated, it resonates with the experiences of our participants because the average age of the FYRNs in our sample was 22 years. Therefore, they were living through late adolescence in their formative years as students of nursing and are still in early adulthood 
now they are FYRNs.

We believe the deductive themes we use to describe the data deepen our analysis and in no way distort the concerns about transitioning to practice expressed to us by the participants.

\section{FINDings}

Thematic analysis enabled us to reduce our FYRN data to three initial descriptive themes and six subthemes. The first descriptive theme, classroom learning, summarized what the participants said they learned from classroom instruction. Two subthemes, learning the optimal and the perfect, and acquiring knowledge rather than skills clarify the participants' perceptions. The second descriptive theme, workplace realities, and its subthemes coping with the burden of work, accepting personal responsibility, learning one's place, and thinking about leaving summarize the challenges FYRNs experience when transitioning to practice. FYRNs are overwhelmed by their workload and disappointed by the low status of nurses compared to medical students and residents. They lament the lack of autonomy in nursing and their dependence on medical orders for routine procedures. The third theme, "wanting a life", summarizes how shift work isolates FYRNs from family and friends and involves thinking about leaving.

The exemplary statements summarized by the themes answer three questions: What did FYRNs learn about nursing from classroom instruction? How do FYRNs describe their experience on clinical units? How are the lives of FYRNs affected by the transition to practice? We will show that FYRNs are troubled by the disparity between learning how nursing should be practiced in the classroom and the really of work pressures in clinical units. FYRNs struggle to maintain a personal life while adjusting to the difference between the world of the classroom and the world of work. After noting that the inductive development of descriptive themes and the deductive development of explanatory themes from pre-existing theory are accepted applications of thematic analysis, ${ }^{[30]}$ we moved from a descriptive to an explanatory level of analysis by adopting the persective of ego-identity theory. ${ }^{[31-33]}$

In this section, we use deductive themes derived from egoidentity theory and the descriptive themes from which they were re-conceptualized to report the findings of the study. We have retained the descriptive themes because we did not set out to apply ego-identity theory and, therefore, did not specifically probe our participants to obtain data on identity formation. For example, we did not ask our participants about how transitioning to nursing practice had affected their sense of self, about how becoming a nurse had affected other people's perceptions of them, or about how the challenges

Published by Sciedu Press they were facing might be life-stage rather than occupationrelated.

\subsection{Theme 1: Forming a nursing identity (initially the- matized as classroom learning)}

The descriptive theme, classroom learning was formed by aggregating data coded as preparation, classroom instruction, learning in the simulation laboratory, knowledge acquisition and skill development. The theme summarizes participants' accounts of what they learned in the classroom, the processes through which they learned, and the relevance to practice of classroom instruction. The theme of classroom learning was conceptualized deductively by recognizing that what the participants were describing was the beginning formation of a nursing identity. Forming a nursing identity means developing a sense of self that fits with nursing ideas and values supported in classroom instruction. As can be seen from the exemplary statements that follow, forming a nursing identity involves internalizing values and nursing knowledge. Although the two learning processes coincide, they are analytically discrete because one engages moral sensitivities, whereas the other involves critical reflection on attaining and applying knowledge. Initiating the formation of a nursing identity that has a moral compass as well as familiarity with the body of knowledge learned in the classroom is fundamental to developing the outlook of a professional nurse.

\subsubsection{Learning the optimal and the perfect}

FYRNs in our sample conceptualized the university as a space that idealizes nursing in a way foreign to everyday practices in clinical settings. As one participant explained, "In the university, they can't teach you how RNs really work because they want you to learn "the ideal way"' (Wadha, P.1). This disparity between the work of the $\mathrm{RN}$ and its idealization in the classroom is central to the challenge of transitioning to nursing practice.

I found a big difference between the program and reality. As soon as I went on the clinical floor as a student, I found out school teaches you the optimal; the best knowledge, everything perfect. In the real world of the clinical units, it is not the same. We were shocked! (Wafaa. P1)

The difference between the nursing classroom and clinical units is stark:

The way RNs work in the unit is different from what we learned at university. We were living in a dream world, trying to learn everything, the optimal things and the perfect things. The reality of nursing is not like this. No one on the 
clinical floors works the way we were taught; the way nursing should be. There is no interaction with patients. The RNs spend minimal time with them. They only finish their paperwork, the basic treatments, and that's it. I work with patients in another way. I spend more time with them. It is a completely different thing, a different approach to nursing, more like what we learned at school. (Wiam, P.2)

Among the things that stand out in these comments are the emphasis on the "optimal" and the "perfect" and the implied humanitarian values seen as dispensed with in the lack of "interaction with patients". "The minimum time spent with patients" is not how it is supposed to be, not what should happen, not what is "optimal", still less something "perfect". We noticed, too, the priority given to paperwork and basic treatments.

FYRNs adapt to the realities of clinical units by blending classroom knowledge with the practicalities of bedside nursing:

They [the RNs in the units] are doing what is practical, what is safe, what we could not learn at university because everything is perfect there. We must listen to the experienced RNs and be guided by them, but without losing our critical thinking and judgment. (Maalam, P.3)

Here the influence of classroom teaching is apparent in the references to "critical thinking" and "judgment" as attributes of the professional nurse, but FYRNs recognize, too, the differences between being a student and being responsible for patients.

At university, we were pampered. Clinical training did not involve actual work. We did not learn that we must be pushed into the water if we are to learn to swim. Our preceptors supervised everything we did. We only observed what was going on and did not do anything except under close supervision. (Wafaa. P.1)

As they transition to nursing practice, FYRN's leave the protection of the classroom for the realities of work. The metaphor of swimming was used with regret, with a sense of not being pushed to cope, of being overprotected; of not being encouraged to take personal responsibility for one's conduct and learning.

\subsubsection{Acquiring knowledge rather than skills}

The participants reported lack of sufficient attention to the development of nursing skills during their undergraduate education. For them, learning at university privileged formal knowledge above the skills required to care for patients.

During the course, we did not master the skills we needed to learn. We focused on knowledge, on what we needed to know, and how we needed to think. In the end, we had to struggle to learn some practical skills because we realized we would need them when we started working with patients. Now we are in the clinical units, the challenge is to learn the skills we should have had before taking on responsibilities for patient care. (Wafia, P.4)

The FYRNs talked about the same emphasis on knowledge rather than skills during clinical rotations. As one participant explained, diagnoses, disease etiology and medications were what was important. Less attention was given to treatment and nursing care.

As students, we were going on clinical rotations of six hours or so. Most of these hours were taken up with gaining knowledge. We were asked, 'What does this medication do?' 'How does it work?' 'What interactions does it have?' Clinical rotations should have emphasized clinical skills not theoretical knowledge we could learn in the classroom. All of us graduated without being able to put in an IV [intravenous line]. (Wakee, P.5)

According to the FYRNs communicating with patients was another neglected learning need.

Skill wise, we needed a lot more involvement with real patients and situations. We had the simulation lab[oratory] and it helped, but real patients react to you and what are doing; it's way different. When we started taking the skills courses, doing a nursing assessment got clearer, but these courses were not as strong as they should have been. There was not enough practice. (Walaa, P.6)

\subsection{Theme 2: Forming an occupational identity (initially thematized as workplace realities)}

The descriptive theme, workplace realities was formed by aggregating data coded as clinical placements, reception by registered nurses, organization of nursing work, nursing activities, medical students, shift work, and workload. This 
theme summarizes participants' accounts of nursing work and discrepancies between classroom learning and nursing practice. The theme was conceptualized deductively by understanding that what the participants were describing was socialization into the world of work. Forming an occupational identity means learning to cope with work demands by immersing oneself in what has to be done, when, how, and with what deference to whom. As can be seen from the exemplary statements that follow, forming an occupational identity involves learning to cope with the physical and psychological demands of work pressures, becoming accustomed to the pace of work, accepting responsibility for one's learning, knowing one's place, and struggling with whether nursing is the right occupational choice.

The ideals and knowledge learned in the classroom are challenged when FYRNs begin work in clinical units. During classroom instruction, students learn to prioritize an ethic of optimal care over the realities of the workplace and the assimilation of information over the acquisition of practical skills. When they start working in the clinical units, they soon realize that getting through the workload on time takes precedence over delivering optimum care.

I feel sorry for myself because I am thinking about leaving bedside nursing. Oh my God! The physical and psychological challenges of the workload are overwhelming. I am sorry for the profession because nursing should be about ethics and caring, but everything from giving five patients to one RN to look after to the allocation of shifts contradicts what nursing should be. (Wasqas, P7)

In the clinical units, the ethic of optimum care is displaced by the realities of practice. "The workload is huge. I do not feel that there is anything that can be done to solve this crisis in workload" (Maalam, P.3). Furthermore, the workload crisis is intensified by inequalities in workload allocation. "[The RNs] push so many tasks to you just because you are new to the floor." (Wakee, P.5)

\subsubsection{Coping with the burden of work}

The high workload takes its toll as FYRNs do their best to cope.

I always come early and start everything before the shift begins. If I am on day duty, I finish on time at 3:00 pm, but without taking a break and without going to the bathroom during the shift. It's really, really, hard. (Muin, P.8)
The pace of work has its effects as well:

We did not expect this workload. I lost 2-3 kilograms during the first month on the unit because I didn't have time to eat at work. I couldn't sleep when I got home because I was too tired (Warda, P.9)

Despite the work pressures, FYRNs try to remain true to the ethic of optimum care. "I feel so overwhelmed physically and mentally. I am really tired, but I don't take breaks because I want to be with my patients". (Wakee, P.5)

\subsubsection{Accepting personal responsibility}

FYRNs explained that they always had a clinical instructor to ask when they needed help or advice, but after graduation they had only themselves to rely on.

When I started on the floor, I realized that there are responsibilities I was not aware of. As a student going to the hospital, there was always an instructor. If there was something missing, I would check with the instructor. If a patient was sick, I would check with the doctors or RNs on the floor. As an RN, there is only yourself. (Wifaq, P.10)

Patients are allocated to you and you are responsible for them. When you are a student you always have support, but when you are an RN you might have a preceptor but it's not the same. You are responsible for your actions because they affect patient safety. (Wabisa, P.11)

\subsubsection{Learning one's place}

The FYRNs talked about status differences between physicians and nurses:

Physicians cause a lot of pressure.

We cannot give an opinion about anything. I read an ECG [electroencephalogram] and had a different opinion to the physician. I was right, but he is privileged because he is a physician and cannot be questioned. This depresses me because no matter how much I study, I will be a nurse who follows doctors' orders (Wadiya, P.13)

FYRNs find out that they do not have the status they thought they would have when they were students.

As new RNs, we have this idea that we are change agents and the leaders of the future. In 
the units, we soon learn that we only carry out orders. I dare not even think about giving my opinion to a physician. (Mahad, P.14)

Partnership in care is an illusion:

I thought I would be a partner in care, but I am only a follower. Some things are purely nursing such as dressing a pressure ulcer. You assess what is required, do the treatment, and document what you have done. But you cannot get started until you have an order from an intern who has not seen the pressure ulcer and does not know anything about dressings. So, we write the order and tell them to sign. I am shocked because no one told us that we have this very narrow scope of practice. It's shocking and disappointing. (Maalam, P.15)

One FYRN talked about "begging" physicians to do something about a patient's pain: "Relationships and communications with physicians are difficult. When patients are in pain, we must beg the physicians to do something for the patient's sake" (Walaa, P.6). However, FYRNs are careful not to cross a physician imposed red line:

I had a patient that needed a tracheostomy. His son asked me about his father. I told him his father was scheduled for a tracheostomy the next day. The son said his father did not want to have surgery. I told him to speak to the physician. The physician was upset and told me, 'It wasn't your job to [tell the relative about the tracheostomy]'. (Moin, P.16)

Gaining acceptance from experienced RNs is another challenge. "No matter what you do or what you accomplish, they are waiting for you to miss something. Everyone monitors you. No matter what, they treat you as if something is missing" (Wafaa, P.1).

When I was a student, the other students were my friends. They would cover up for me and I would cover up for them. Now I am a RN, I work with other RNs. Some gossip about you, others defend you, some try to hurt you. (Wafia, P.4)

\subsubsection{Thinking about leaving}

The FYRNs commented on reality shock and thinking about leaving:

For me, the transitional period between student and becoming a registered nurse was the most devastating period in my life. I had reality shock to the extent I wanted to quit. I went and told them, 'I don't want this anymore'. Thinking back, I was not prepared well enough to be a nurse (Wasiba, P.11)

And:

We rotated through all the units and different specialties as students, but we did not see the magnitude of the workload. If I had seen things the way they really are, I would have thought twice about continuing my nursing studies. I am still thinking about leaving. (Maalam, P.3)

\subsection{Theme 3: Realizing a cultural identity (initially the- orized as wanting a life)}

The descriptive theme, "wanting a life" was formed by aggregating data coded as working nights, weekend work, working on public holidays, called in from off duty, family and friends. The theme summarizes how working hours impact the ability to achieve work-life balance. The theme was conceptualized deductively by understanding that what the participants were describing was the impact of nursing work on the ability to meet the expectations of family and friends. Realizing a cultural identity means trying to maintain a personal life and meet the expectations of family and friends while working unsocial hours and feeling exhausted by the pressures of work. As can be seen from the exemplary statements that follow, realizing a cultural identity involves struggling to maintain relationships and achieve a sustainable work-life balance. Lebanon has a collective culture in which family ties are strong and many families live in multi-generational households. Young people are under immense pressure to live within the bosom of their families while enjoying a gregarious life-style with their peers. The many religious festivals in the country add to the pressures on young people to spend time with their parents, siblings and extended families. There are few weekends when families living in Beirut do not go to the mountains to spend time with relatives and friends. During the week, there is a lively clubbing culture that Lebanese young people enjoy. Accordingly, work-life balance is a challenge for FYRNs, especially if they have not experienced shift work as a student.

My problem is not the clinical floor or the physicians. It's my life and I want it back. I want to see my friends. It has been months since I have seen them. Having a personal life is my challenge. (Warda, P. 9) 
Time management is perceived to be at the core of the problem of work-life balance: "Time management is the issue. Managing your time on duty, managing your off-duty time, making time for leisure, finding time to sleep; organizing your whole life" (Wafia, P.4).

Night duty is especially challenging.

When you work night shifts, you are the opposite the entire world. You do not see your family. When I am sleeping, they are awake; when they are sleeping, I am awake, or at work. I do not see my family much and my friends not at all. Rotating shifts between nights and days is a real challenge. (Wakee, P.5)

The work-life balance pressures on FYRNs are intensified by the priority given to the needs of experienced RNs.

I asked for a holiday to prepare for my engagement. They said you can take a vacation when you have worked a year. I didn't want a vacation, I wanted a holiday. They said, 'No'. More experienced RNs are given holidays for their university work, but I can't take a holiday for my engagement. (Maalam, P.15)

\section{Discussion}

FYRNs are troubled by the differences they find between the ideals and protocols they learn in the classroom and the realities of nursing practice. The first of our descriptive themes places more emphasis on the optimal perspective that informs classroom instruction than found in previous studies and highlights once again the perception that insufficient skills are acquired during baccalaureate nursing education. The second descriptive theme and its subthemes add to the literature on the burden of coping with nursing work while continuing to learn during the difficult process of transitioning to practice. The third theme, preserving cultural identity, highlights the challenges FYRNs in collective cultures face in achieving an acceptable work-life balance. Whereas, all FYRNs experience similar challenges with shiftwork and weekend work, the pressures on young people to satisfy family expectations are exceptionally strong in Lebanon and other countries with collective cultures. Family expectations of young unmarried women are especially demanding. ${ }^{[34,35]}$

All three themes relate to the sense FYRNs have of self as they transition to adulthood as well as to nursing practice. In Erikson's theory of human development, ${ }^{[31,32]}$ a person's identity is developed in age-related stages that involve successful resolution of personal crises that arise from the interplay of psychological, cultural and socio-historical factors.
For FYRNs, as for others of the same age, independence becomes important when transitioning to new roles and relationships. The transition to practice involves examining personal values and setting goals while responding to demands of new roles. According to Marcia, ${ }^{[33]}$ the formation of an occupational identity involves committing to a specific profession or line of work by making a personal decision after considering meaningful alternatives. Until a choice has been made, the FYRN like any other young person is in one of two intermediate psychological states between uncertainty and settled occupational choice.

Moratorium is the term Marcia uses to describe the psychological state of young people who are unable to reconcile what they believe in and as a result are incapable of committing to an occupation. Conversely, a young person in foreclosure has yet to experience the predicament of moratorium. Marcia explains that foreclosure involves "a certain rigidity" ${ }^{[33]}$ in which the young person feels extremely threatened in situations that challenge existing beliefs and ways of functioning.

The continuum described by Marcia ${ }^{[33]}$ provides a way of thinking about the transition to nursing practice. The process involves reconciling an emergent professional nursing identity nurtured in the classroom with an occupational identity that fits the requirements of clinical units, while at the same time trying to preserve a cultural identity ruptured by the demands of the workplace.

FYRNs in an identity moratorium are likely most at risk for leaving because their commitment to nursing is at best equivocal as they face up to the challenges of transition. The FYRN in foreclosure is more likely to experience the transition to nursing practice as a threat to existing values and beliefs. The transition to nursing practice is likely to be particularly arduous for those FYRNs who were strongly attracted to nursing prior to their university studies because the values that bind them to nursing are radically challenged by the realities of the workplace. What Marcia regards as a certain rigidity in beliefs and ideals reinforced by undergraduate nurse education, makes these FYRNs extremely vulnerable to the stress of transitioning to practice.

Ego-identity theory indicates that the transition to nursing practice is not the same for all FYRNs. For those who are still to form an occupational commitment, their relationship to nursing is at best tenuous and easily abandoned, especially if alternative and potentially more satisfying employment opportunities are available. Those FYRNs in moratorium are next in vulnerability to leave nursing, subject to the availability of other career options to explore. In the short to medium term, FYRNs in foreclosure are more likely to 
stay, but at considerable emotional and personal cost. Eventually FYRNs in foreclosure will experience the crisis of moratorium and become vulnerable to leaving nursing as they come to understand themselves and make an informed choice about their career preferences. Therefore, strategies specific to the complexities of identify formation are required if FYRNs in Lebanon are to be supported to remain in the nursing workforce. Recent literature on identity formation supports out interpretation. Identity formation is still seen as the fundamental challenge of transitioning from adolescence to adulthood. The construction of identity is central to how individuals define themselves in organizations, ${ }^{[36,37]}$ and the central challenge in enacting multiple identities. ${ }^{[38]}$ Accordingly, theories of identity formation offer considerable theoretical scope for understanding why the transition to nursing practice is so difficult for FYRNs. Our emphasis on identity formation as a major psychological process in the transition to nursing practice complements the literature we reviewed above which focuses attention more centrally on contextual variables that affect occupational transition experiences. The challenge for the future is to understand the interactions between the psychological, organizational and societal factors involved to better understand how to facilitate the construction and maintenance of occupational identities. ${ }^{[39]}$

\section{Limitations}

The study has important limitations. The views of focus group members might not have been representative of those of all the FYRNs who graduated with them from the participating universities. It is possible that the FYRN's who consented to take part in the study were experiencing more challenges than usual for FYRNs making the transition to nursing practice in Lebanon. Since all the participants were recruited from three of Lebanon's leading universities, it is possible that their experiences, however, challenging, were less difficult than those experienced by nurses graduating from other universities; that is, we may have underestimated the challenges FYRNs face in Lebanon. Furthermore, we did not conduct focus group discussions with registered nurses on the clinical floors who receive FYRNs, with preceptors, nurse managers, nurse leaders, nurse managers, faculty or clinical instructors. Therefore, our study focused on the transition to nursing practice from the perspective of only one of the interested parties.

\section{Conclusion}

FYRNs in Lebanon are struggling to transition successfully to the role of registered nurse. According graduates of three of Lebanon's leading universities, preparation in the classroom emphasizes optimal practice that is at odds with the way nursing is practiced in clinical units. The burden and pace of work overwhelms new graduates and they are at risk for becoming disillusioned when they are not made to feel welcome and not treated fairly by experienced registered nurses. The struggle to keep up with the workload of a registered nurses, leaves FYRNs at risk for leaving nursing, especially if they are ambiguous about their career choices and prospects. The life styles around family and friends expected in a collective culture are ruptured by the demands of working shifts and especially night duty. More studies are needed to further examine the transition to nursing practice in Lebanon and other countries in the Eastern Mediterranean region. We hope our findings will encourage more investigators to theorize the themes they identify in qualitative studies of the transition to nursing practice. Our interpretation of the challenges faced by FYRNs from the perspective of egoidentity theory strongly suggests that strategies are needed to help new graduates cope psychologically with the demands of nursing practice.

\section{ACKNOWLEDGEMENTS}

We would like to thank the FYRNs who took time to attend our focus group discussions for sharing their experiences, concerns, and aspirations for nursing so openly and patiently.

\section{CONFLiCTS OF INTEREST Disclosure}

We individually declare no conflict of interest relevant to our study or this article.

\section{REFERENCES}

[1] Lee H, Hsu MT, Li PL, et al. 'Struggling to be an insider': a phenomenological design of new nurses' transition. J Clin Nurs. 2013; 22(5-6): 789-97. https ://doi .org/10.1111/j.1365-2702. 20 12.04189. $\mathrm{x}$

[2] Rafii F, Sajadi Hezaveh M, Seyedfatemi N, et al. Suffering sources among the newly-graduated nurses at the beginning of their clinical work: a qualitative study. Life Sci J. 2012; 9(4): 5517-24. PMid:26032056.

[3] Erickson SK. Mentorship programs and the novice nurse: a rapid evi- dence [master's thesis]. Vancouver (BC, CA)]: University of British Columbia; 2015; 139 p.

[4] Horsburgh D, Ross J. Care and compassion: the experiences of newly qualified staff nurses. J Clin Nurs. 2013; 22(7-8): 1124-32. PMid:23480502 https ://doi.org/10.1111/jocn. 12141

[5] Jewell A. Supporting the novice nurse to fly: a literature review. Nurse Educ Pract. 2013; 13(4): 323-327. PMid:23643823 https : //dx.doi.org/10.1016/j.nepr.2013.04.006

[6] Pfaff K, Baxter P, Jack S, et al. An integrative review of the fac- 
tors influencing new graduate nurse engagement in interprofessional collaboration. J Adv Nurs. 2014; 70(1): 4-20. PMid: 23815377 https://doi.org/10.1111/jan.12195

[7] Shrestha S, Joshi S. Lived experiences of staff nurses during first six months of their employment in a university hospital. J Nepal Health Res Counc. 2014; 12(28): 182-186. PMid: 26032056

[8] Howard-Brown C, McKinlay E. Aged residential care nurse entry to practice (ARC NETP) program evaluation final report. Wellington, [NZ]: Sapere Research; 2014. Summary. Available from: http://www .health.govt.nz/publication/aged-residen tial-care-nurse-entry-practice-arc-netp-programme -evaluation-report [Accessed: 28 July 2017].

[9] Mellor P, Gregoric C. Ways of being: Preparing nursing students for transition to professional practice. J Contin Educ Nurs. 2016; 47(7): 330-340. PMid: 27351266 https ://doi.org/10.3928/002201 24-20160616-10

[10] Phillips C, Kenny A, Esterman A, et al. A secondary data analysis examining the needs of graduate nurses in their transition to a new role. Nurse Educ Pract. 2014; 14(2): 106-111. https: //doi.org/10.1016/j.nepr.2013.07.007

[11] Idvall E, Ekström L. Being a team leader: newly registered nurses relate their experiences. J Nurs Manag. 2015; 23(1): 75-86. PMid:2382289 https://doi.org/101111/jonm12085

[12] Sparacino LL. Faculty's role in assisting new graduate nurses' adjustment to practice. SAGE Open Nurs. 2016; 2: 1-9. http: //dx.doi.org/10.15640/ijn.v2n2a5

[13] Parker V, Giles M, Lantry G, et al. New graduate nurses' experience in their first year of practice. Nurse Educ Today. 2014; 34(1): 150156. PMid: 22857819 https://doi.org/10.1016/j.nedt. 201 2.07 .003

[14] Carnevale AP, Smith N, Gulish A. Nursing: supply and demand through 2020. Georgetown (Washington, DC) Georgetown University, Center on Education and the Workforce. 2015; 47 p. Available from: https://cew.georgetown.edu/wp-content/upl oads/Nursing-Supply-Final .pdf [Accessed: 24 July 2017].

[15] El-Jardali F, Alameddine M, Dumit N, et al. Nurses' work environment and intent to leave in Lebanese hospitals: implications for policy and practice. Int J Nurs Stud. 2010; 48(2): 204-214. PMid: 20932524 https://doi.org/10.1016/j.ijnurstu.2010.07.009

[16] El-Jardali F, Alameddine M, Jamal D, et al. A national study on nurses' retention in healthcare facilities in underserved areas in Lebanon. Hum Resour Health. 2013; 11-49. PMid: 24079458 https://doi.org/0.1186/1478-4491-11-49

[17] Spence Laschinger HK, Zhu J, Read E. New nurses' perceptions of professional practice be behaviours quality of care job satisfaction and career retention. J Nurs Manag. 2016; 24(5): 656-65. PMid: 26932145 https : //doi.org/10.1111/jonm. 12370

[18] Fielden JM. Managing the transition of Saudi new graduate nurses into clinical practice in the KSA. J Nurs Manag. 2012; 20(1): 2837. PMid: 22229898 https://doi.org/10.1111/j.1365-283 4.2011.01348.x

[19] Kramer M, Brewer BB, Maguire P. Impact of healthy work environments on new graduate nurses' environmental reality shock. West J Nurs Res. 2013; 35(3): 348-383. PMid: 21498813 https : //doi.org/10.1177/0193945911403939

[20] Boehm LB, Tse AM. Application of guided imagery to facilitate the transition of new graduate Registered Nurses. J Contin Educ Nurs. 2013; 44(3): 113-119. PMid: 23330588 https://doi.org/10.3 928/00220124-20130115-16

[21] Nematollahi R, Isaac JP. Bridging the theory practice gap: a review of graduate nurse program (GNP) in Dubai, United Arab Emirates. Int Nurs Rev. 2012; 59(2): 194-9. PMid:22591090 https: //doi.org/10.1111/j.1466-7657.2011.00949.x
[22] Chênevert D, Jourdain G, Vandenberghe C. The role of highinvolvement work practices and professional self-image in nursing recruits' turnover: a three-year prospective study. Int J Nurs Stud. 2016; 53: 73-84. PMid: 26421911 https://doi.org/10.1016/ j.ijnurstu.2015.09.005

[23] Tastan S, Unver V, Hatipoglu S. An analysis of the factors affecting the transition period to professional roles for newly graduated nurses in Turkey. Int Nurs Rev. 2013; 60(3): 405-412. PMid: 23961804 https://doi.org/10.1111/inr.12026

[24] Zarshenas L, Sharif F, Molazem Z, et al. Professional socialization in nursing: a qualitative content analysis. Iranian J Nurs Midwifery Res. 2014; 19(4): 432.

[25] Watt E, Pascoe E. An exploration of graduate nurses' perceptions of their preparedness for practice after undertaking the final year of their bachelor of nursing degree in a university-based clinical school of nursing. Int J Nurs Pract. 2013; 19(1): 23-30. PMid: 23432885 https://doi.org/10.1111/ijn.12032

[26] D'ambra AM, Andrews DR. Incivility retention and new graduate nurses: an integrated review of the literature. J Nurs Manag. 2014; 22(6): 735-742. PMid: 23927565 https://doi.org/10.1111/ jonm. 12060

[27] Leong YM, Crossman J. New nurse transition: success through aligning multiple identities. J Health Organ Manag. 2015; 29(7): 1098-1114. PMid: 26556170 https://doi.org/10.1108/JHOM -02-2014-0038

[28] Langford BE, Schoenfeld G, Izzo G. Nominal grouping sessions vs focus groups. Qual Market Res Int J. 2002; 5(1): 58-70. https: //doi.org/10.1108/13522750210414517

[29] Tuckett AG. Qualitative research sampling: the very real complexities. Nurs Res. 2004; 12(1): 47-61. PMid: 15493214 https: //doi.org/10.7748/nr2004.07.12.1.47.c5930

[30] Braun V, Clark V. Using thematic analysis in psychology. Qual Res Psych. 2006; 3(2): 77-101. Available from: http://eprints . uwe . ac . uk/11735 [Accessed: 9 December 2016]

[31] Erikson EH. Childhood and society. New York (NY): Norton; 1956; 1953-1993 p.

[32] Erikson EH. The problem of ego identity. J Am Psychoanal Assoc. 1956; 4(56): 56-121. PMid:13286157 https ://doi.org/10.117 7/000306515600400104

[33] Marcia ME. Development and validation of ego-identity status. J Am Psychoanal Assoc. 1966; 3(5): 551-588.

[34] Sidani YM, Al Hakim ZT. Work-family conflicts and job attitudes of single women: a developing country perspective. Int J Hum Resour. 2012. http://doi.org/10.1080/09585192.2011.579919

[35] Jabbra NW. Family feast observance in a Lebanese Melkite village. Middle East Crit. 2016; 25(2): 147-161. http://dx.doi .org/10. 1080/19436149.2016.1141586

[36] Ashforth BE, Schinoff BS. Identity under construction: how individuals come to define themselves in organizations. Ann Rev Org Psych Org Behav. 2016; 3: 111-137. https ://doi .org/10.1146/annu rev-orgpsych-041015-062322

[37] Brown AD. Identities and identity work in organizations. Int J Manag Rev. 2015; 17(1): 20-40. https ://doi .org/10.1111/ijmr. 120 35

[38] Ramarajan L. Past present and future research on multiple identities: toward an intrapersonal network approach. Acad Manag Ann. 2014; 8(1): 589-659. https://doi.org/10.1080/19416520.2 014.912379

[39] Brouard F, Bujaki M, Durocher S, et al. Professional accountants' identity formation: an integrative framework. J Bus Ethics. 2017; 142(2): 225-238. https://doi.org/10.1007/s10551-016-3 $157-z$ 